UDC 633.14:631.53/532.

(C) 2018

\title{
Principles and methodology of takeoff in the system of selection of traditional crops of Polissia region
}

\author{
Chernuskyi V. \\ candidate of agricultural sciences \\ Institute of agriculture of Polissia region of NAAS
}

The purpose. To find identical analytic forms of parametric behavior of component attributes in the system of ontogenetic development and in the system of disjoining generations for construction of prognostic trends of takeoff and identification of attributes perspective for selection by results of one ontogeneticvegetative period. Methods. Analysis of geometrical properties of evolutionary distances of selection system of component attributes as mathematical plural. Results. Identical analytic forms of parametric behavior of component attributes in the system of ontogenetic evolution and in the system of generations which disjoin are detected in different crops in selection seed beds. During differentiation of these systems two systems of interaction of mainly linear and non-linear character are detected. Conclusions. At the first stage of selection the most effective is takeoff in linear system by the principle of optimization of vectorly gradient summations of orthogonal projections of parameters of component attributes. At the second stage of selection in the system of multifactorial interconditionality of attributes according to additive-multiplicative principles it is necessary to use non-linear methods of analysis in phase-parametrical space with the purpose of selection of bifurcations, basins of attraction, solitons, etc. for forming synergistic systems of takeoff.

Key words: takeoff, winter rye, field pea, component attributes, linear analysis, non-linear analysis.

A selection is most responsible in the plan of increase of efficiency that energy- labour- and by the financially expense element of plant-breeding process. The unoptimum tuning of algorithm and erroneous determination of paradigm and model of selection can result in a zero competitive result even at the high selfreactance values of initial forms.

On of this time at and plant-breeding selection the system of linear analysis, gene central system of establishment of of inheritance of signs and temporal protracted principle of analysis of markovie processes of microevolution, is used on sentinel rows. This system is an extraordinarily expense in opinion of academician V.A. Dragavceva ineffective [1-3]. In of addition academicians of of Д. Grodzinskiy, V.Glazko mark, that one-sided structural approach, which orients on manipulation separate genes which encode of "Signs with certain functions», and examines ontogenesis as their «register», enables to untie only small, thus far the most not main part of modern plant-breedings tasks [4].

But of in the environment of leading selectionists and geneticists conception of application also of epiegenetic principles is formed in the selection of agricultural cultures, as an inalienable administrative element at the boundary states of genotypes. In of particular in opinion of of S.I. Maleckogo of and academicians of of M.V. Roika of and of V.A. Dragavceva [5,6,7] of consideration of methods of formation of seed testifies at different kinds and births, that mostly hardness to take the genesial signs of plants to Mendel, and their inheritance, as and rule, has epiegenetic nature. Consequently, with the third, epiegenetic and type changeabilities at plants are of CPLD of systems of recreation of seed and morphogenic processes, especially origin of different types of symmetry and fractal structures (as known, they will be realized by of independent organization of and not controlled directly genes). 
But most a significant problem in the technological process of selection is made by a temporal factor. Within the framework of decision of this analytically difficult dilemma of P.P. Litunom with coauthors [8] is offer the system of selection on macrosigns, which includes in particular the system of analysis of them ontogenetic development on the sentinel rows of prediekte on the plural of nonlinear phase-self-reactance space.

Methodological basis of conception is made by the innovative paradigm of academician V.A. Dragavceva with coauthors [9] about coincide displays of ontogenetic networks of organism and phyllogenetic processes of inheritance of gene networks of population. In other words ontogenetic activity of gene networks of organism is examined as a snapshot is adequate phyllogenetic activity of gene networks of population which fission. On this platform the new going is offered near the express estimation of genetics and genetic (additive) dispersions of properties of the productivity of plants.

By us conception of fullgenes of system selection, which includes for itself the analytical platforms of theory of the determined chaos, authentication of genome, is developed in development of these ideas, as a functional «black box» under the management of external factors, and adequate homeomorphic reflection of phenotiepe, as systems of signs on the plural of nonlinear phase-self-reactance space [10-12].

Thus the purpose of our researches is an exposure of are identical of analytical forms of self-reactance conduct of component signs in the system of ontogenetic development and in the system of generations which fission, for the construction of forecast trend of selection and authentication of signs perspective for a selection as a result of one ontogenetic-vegetation period.

Method of researches. In quality methodological basis principles of analysis of geometrical properties are utillized evolution distances [13] for determination of the plant-breeding system as mathematical plural which provides possibility of placing of elements of plural $X$ in some geometrical space and parcel of land of points of this great number by co-ordinates in this space. It, in same queue, allows to apply all of arsenal of methods of multidimensional analysis for research of correlation of internal- and interspecific changeability, visualization of possible directions of evolution, association of these different types, for example molecular and morphological, and estimation of their identical.

In accordance with basis of design of the biological and ergatic systems [14] the general issue of classification of plural of the possible states of the biological or ergatic systems is grounded - BES. An optimum language for such description is a language of theory of plurals and topology: indeed, every state is the class of descriptions of, which characterizes some plural of external influences (for example, plural of change of managing parameters).

We formed the standard surfaces of the additive and additive -multiplcative systems as a mathematical abstract model of additive sumation factors in the most general view of $z=x+y$ for which characteristic unambiguity linearness of one variation decisions (рис.1a) and complete model of sumation of additive action and multiplicative co-operation of factors of $z=x+y+x 2+y 2+x^{*} y$ for which characteristic ambiguousness of decisions of multicollineariti (draw.1b).

Results of researches. As a result of analysis of self-reactance intercommunications of component signs of the productivity of plant at different cultures (diaper, rye, lupin narrow-leaved, potato) in nurseries different degree of plant-breeding processing on the different stages of ontogenetic development of plants formed statisticsliberi surfaces as $3 d$ - configuranion, which represent these copulas in different combinations. Visual differentiation and authentication of analytical surfaces in relation to standards testifies to the presence at least two classes of these systems, namely linear (additive in mathematical sense) and nonlinear (additive multiplicative quadratic also in mathematical sense). 


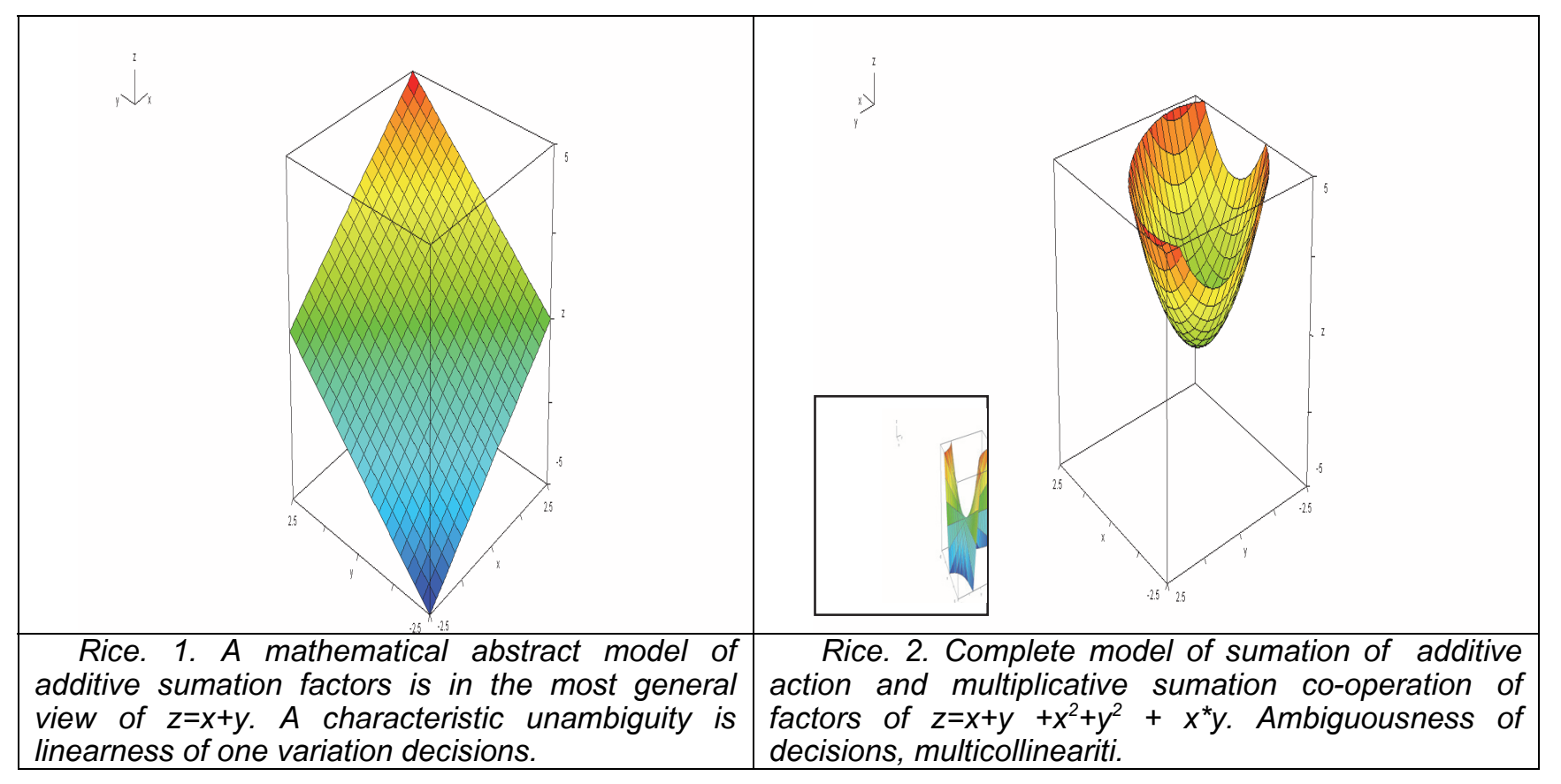

In particular found out linear character of dependences between component signs on the example of standards of rye winter-annual on signs amount of grains in an ear (KZK), mass 1000 grains (MTN), mass of grains from to the ear (MZK). On the first stages of selection linear character of transgressive additive - cluster co-operation presents considerable interest and is a favourable object for a selection. For example in this system of selection of elite ears (on principle of ortogonal optimum vectorial mutual connektiviti of component signs) for period of 2011-2016pp. we attained growth of middle indexes of VZK from 1,67 to 2,63 grammes, KZK - from 55 to 63 things, MTN - from 31r to 44 grammes. Maximal indexes arrived at for VZK from 3,2 $\ulcorner$ to a 5,1 gramme, at KZK - 86 and 103 pieces and MTN - 63,7 and 64,1 grammes accordingly (rice. 2a,б).

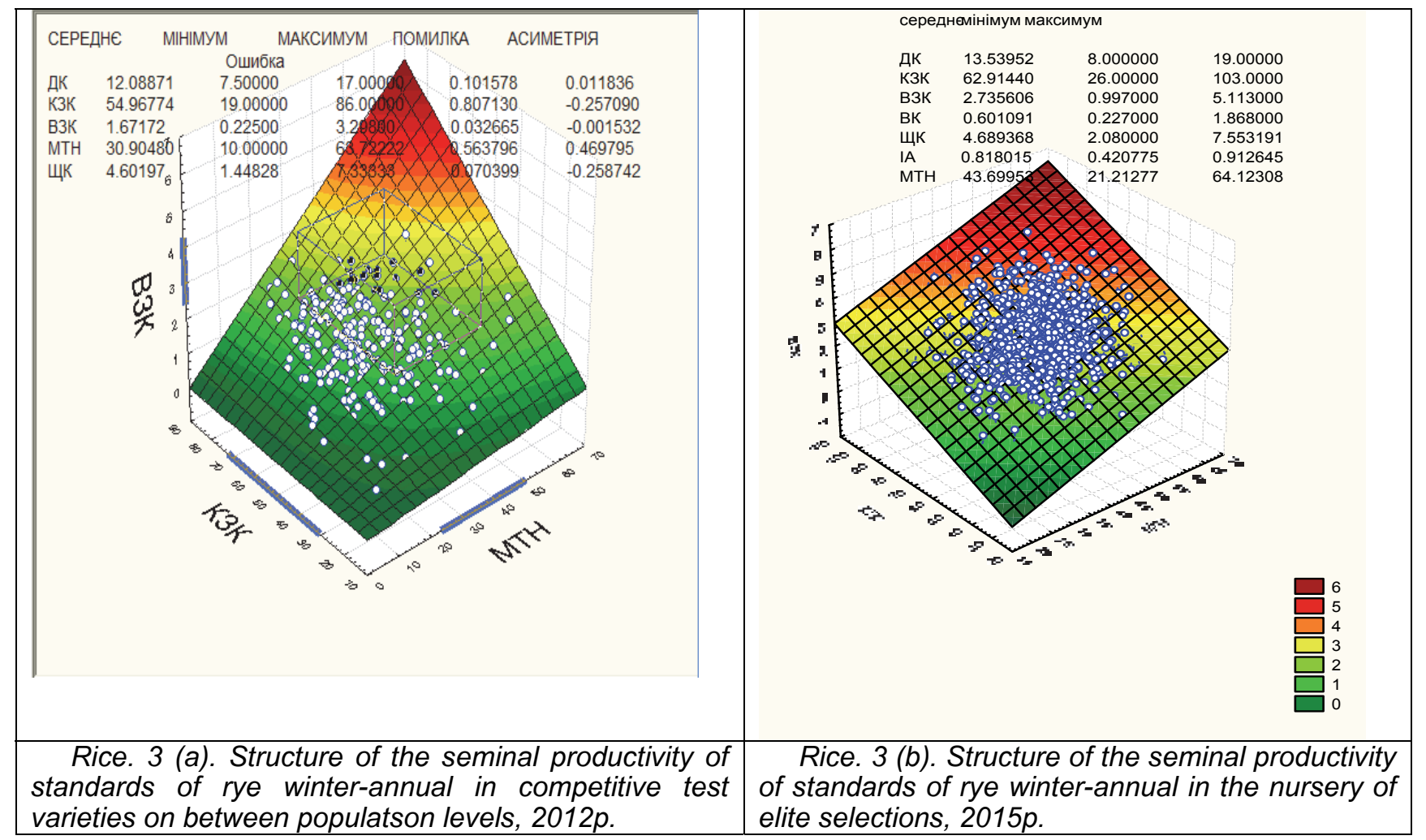


Usually applied by us above all things the algorithms of selection are extraordinarily simplified in relation to organizationally evolutional complication of the biological systems of reproduction of plants and practically exhausted itself for the subsequent use in the plant-breedings programs. Coming from that in obedience to modern innovative presentations population of sort is opened difficult system which independentliorganized in phase-self-reactance space in a that number on principle of feed-back and also, that to these systems above all things inherent emergence, that more simple elements have appearance of new properties of missing, in quality innovative and adequate by us application of method of nonlinear analysis is begun. We see him perspective for subsequent researches with the purpose of increase of efficiency of the plant-breedings programs. A theoretical platform of method is ecsponent of Lyapunova, equalization of Lorenca, Lotki-Vol'teri and others like that, so as for them characteristic branching and the discrete sinergistical states are certain as strange pool of graviti.

In particular we are analyse the phase-self-reactance portrait of the system of microevolution of lines of diaper on the sign of MTN including under the action of ecologicalgradients factors and found out separatision for differentiation of standards in accordance with directions of the economic use. And in the system of crossing wanders on the difficult curvilinear surfaces of hyper complecs multivariable canonical (coefficient got at the decision of real hyper complecs matrices) the models of the system of intercommunications of component signs of plant-breedings standards of rye of winter-annual on ecologicalgradients backgrounds external environment are found synergistik optimized parameters of their combination for a selection. Information futurism difficult prognostic platforms present considerable interest and are perspective for subsequent researches.

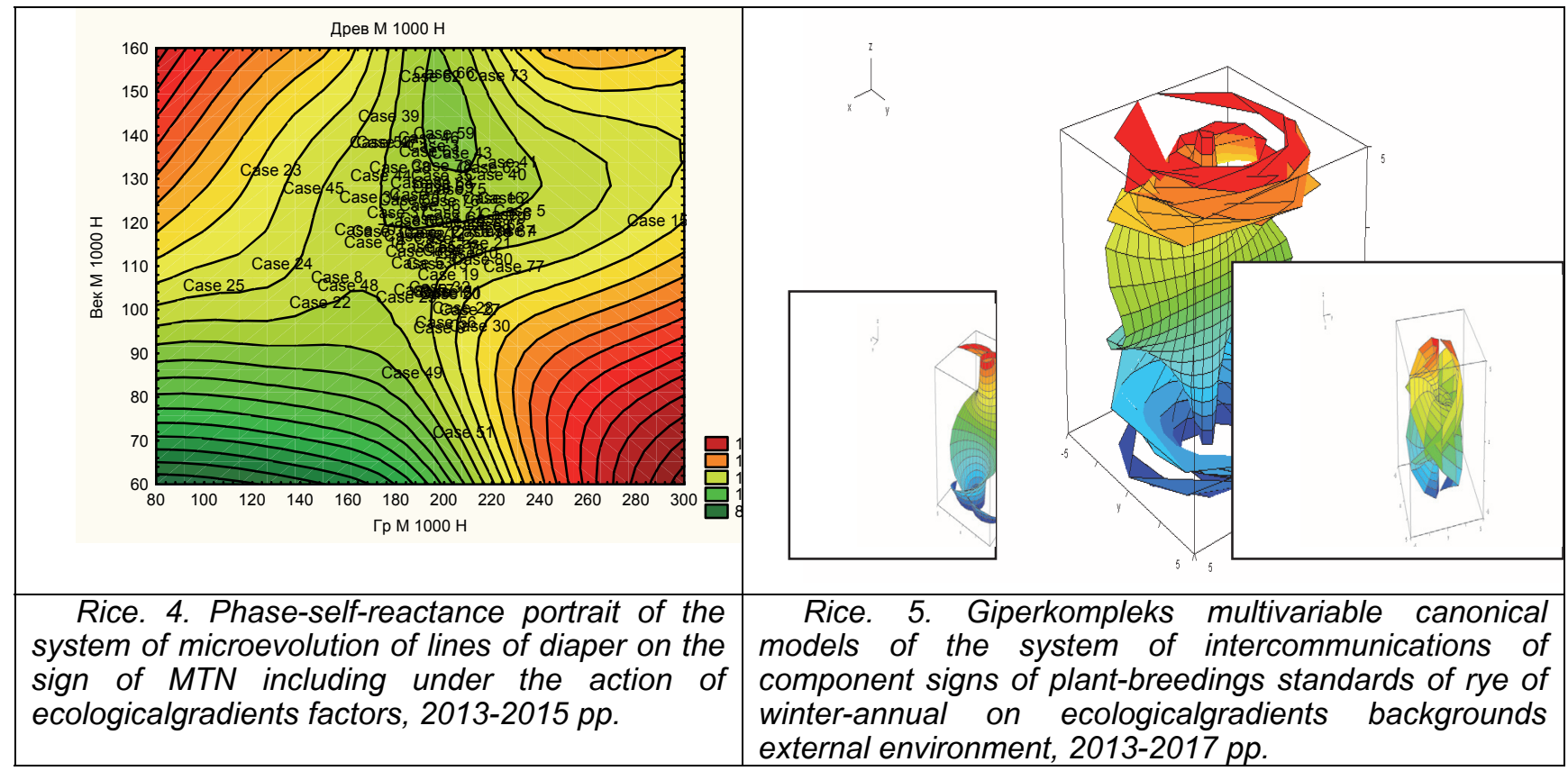

\section{Conclusions}

As a result of structural analysis of the systems of intercommunications of component signs at forming of complex at rye winter-annual, diaper and potato, in the plant-breedings nurseries of different hierarchical level and different years found out the identical analytical forms of self-reactance conduct of component signs in the system of ontogenetic development and in the system of generations which fission

In the process of differentiation of these systems found out two systems of co-operations of mainly linear and nonlinear character. 
On the first stages of selection most effective is a selection in the linear system on principle of optimization of vectorial-gradient sumation of ortogonal projections of parameters of component signs.

On the second stage of selection in the system of multivariable intercommunications signs after additive multiplicative principles application of nonlinear methods of analysis is needed in phase-self-reactance space with the purpose of selection of branching, pools of attraction, солітонів, and others like that for forming of the sinergistical systems of selection.

\section{Bibliography}

1. Dragavcev V.A. (2009). Problemy overcoming of breaks between genes and signs in a modern selection. Information of the Moscow agricultural academy the name of to To. And. Timiryazeva. Moskva. Vyp. 2. P. 110-122. [In Russian].

2. Dragavtsev V.A. (2000). Integration of of Biodiversity of and of Genom Technology for of Crop Improvement. National Institute of of Agrobiological Resources. Japan. Tsucuba. P. 93-95.

3. Dragavtsev V.A. (2002). Algorithms of of an ecology - genetical survey of of genofond and methods of creating the varieties of crop plants for yield, resistance and quality. VIR, St.-Petersburg. $41 \mathrm{p}$.

4. Grodzinskiy D., Glazko V. (2005). Ekological-genetic priorities of intensification of plant-grower. Bulletin. NAN of Ukraine. № 9, P. 57-62. [In Ukrainian].

5. Maleckiy S.I., Roïk N.V., Dragavcev V.A. (2013). Third changeability. Types of heredity and reproduction of seed at plants. Agricultural biology. Problems, reviews. № 5. - P. 3-29. [In Russian].

6. Maleckiy S.I., Maleckaya E.I., Yudanova S.S. (2011). Apozigotiches method of reproduction of seed in the system of sort of Beta (Chenopodiaceae) and homology rows of N.I. Vavilova. Vavilovskiy magazine of genetics and selection. T. 15. № 1. P. 66-79. [In Russian].

7. Maleckiy S.I. (2010). Heredity and synergetics processes at pollination and impregnation at floral plants. Avtokhtonni that introduced plants. Issue 6. P. 90-106. [In Russian].

8. Litun P.P., Kirichenko V.V., Petrenkova V.P. et al (2004). Theory and practice of selection on macro signs. Metodological problems. Kharkiv. 130 p. [In Ukrainian].

9. Dragavcev V.A., Makarova G.A., Kochetov A.A. et al (2012). The new going near the express estimation of genotypic and genetic ( additive) dispersions of properties of the productivity of plants. Vavilovskiy magazine of genetics and selection. T. 16, № 2. P. 427-436. [In Russian].

10. Chernuskiy V.V. (2014). The Methodological going near creation of the system automated complexes of collection and analysis of data in the process of selection in connection with the selection of agricultural grops. Scientific labours of Institute of biotpower cultures and sugar beets. Kyiv, Vip. 22. - C. 54-61. [In Ukrainian].

11. Efimov V.M., Mel'chakova M.A., Kovaleva V.Yu. (2013). Geometric properties evolutionari distances. Vavilovskiy magazine of genetics of breeding. T. 17. - № 4/1. - C. 714-723. [In Russian].

12. Shiyan A.A. (2008). Bases of design of the biological and ergatic systems. The Train aid. Winnitca: VNTU. 131 p. [In Ukrainian]. 